\title{
WEIGHTED INEQUALTIES FOR THE DISC MULTIPLIER
}

\author{
KENNETH F. ANDERSEN ${ }^{1}$
}

\begin{abstract}
ABSTRACr. Charles Fefferman has shown that the disc multiplier is not a bounded operator on $L^{p}\left(\mathbf{R}^{n}\right), n>1, p \neq 2$. On the other hand, Carl Herz has shown that when this operator is restricted to radial functions in $\mathbf{R}^{n}$, it is bounded in $L^{p}\left(\mathbf{R}^{n}\right)$ provided $p$ satisfies $2 n /(n+1)<p<2 n /(n-1)$. In this paper, sufficient conditions on the weight function $\omega$ are given in order that the disc multiplier restricted to radial functions should be bounded in $L^{p}\left(R^{n}, \omega(|x|)\right)$. When applied to power weights $\omega(r)=r^{\alpha}$ these conditions are also necessary.
\end{abstract}

1. Introduction and statement of results. Let $\hat{f}$ denote the Fourier-Plancherel transform of $f \in L^{2}\left(\mathbf{R}^{n}\right)$ and then define the disc multiplier operator $T_{n}$ on $L^{2}\left(\mathbf{R}^{n}\right)$ by

$$
\left(T_{n} f\right)^{\wedge}(x)= \begin{cases}\hat{f}(x) & \text { if }|x|<1 \\ 0 & \text { if }|x|>1\end{cases}
$$

It is well known that $T_{1}$ extends to a bounded linear operator from $L^{p}(\mathbf{R})$ to itself if $1<p<\infty$ but that for $n>1, T_{n}$ is bounded on $L^{p}\left(\mathbf{R}^{n}\right)$ only if $p=2$. This last fact is C. Fefferman's celebrated negative resolution of the disc conjecture [2]. On the other hand, I. Hirschman [4] has shown that $T_{n}$ is bounded on the power weighted spaces $L^{2}\left(\mathbf{R}^{n},|x|^{\alpha}\right)$ if $-1<\alpha<1$.

If $f(x)$ is radial, $f(x)=f_{0}(|x|), x \in \mathbf{R}^{n}$, then $\left(T_{n} f\right)(x)$ is also radial and we write $\left(T_{n} f\right)(x)=\left(R_{n} f_{0}\right)(|x|)$. C. Herz [3] proved that $T_{n}$ restricted to radial functions is bounded in $L^{p}\left(\mathbf{R}^{n}\right)$ provided $2 n /(n+1)<p<2 n /(n-1)$. This range for $p$ is best possible and in fact, as C. Kenig and P. Tomas [5] have shown recently, this operation is not of weak type $(p, p)$ for $p=2 n /(n+1)$ or $p=2 n /(n-1)$.

The purpose of this paper is to prove certain weighted analogues of the result of Herz. We shall give sufficient conditions on a nonnegative weight function $\omega(|x|)$ so that $T_{n}$ restricted to radial functions is bounded in $L^{p}\left(\mathbf{R}^{n}, \omega(|x|)\right)$. When applied to power weights, $\omega(|x|)=|x|^{\alpha}$, the sufficient conditions are also necessary. Our main result is the following theorem.

Received by the editors September 26, 1980. The results contained in this paper were announced at the Summer Seminar on Harmonic Analysis of the Canadian Mathematical Society held at McGill University, Montreal, August 1980.

1980 Mathematics Subject Classification. Primary 42B15.

Key words and phrases. Disc multiplier, radial functions, spherical harmonics, weighted inequalities.

${ }^{1}$ Research supported in part by NSERC grant \#A-8185. 
THEOREM 1. Let $1<p<\infty, 1 / p+1 / p^{\prime}=1$ and suppose $\omega(r)$ is a nonnegative function defined on $(0, \infty)$ for which there is a constant $K$ such that

$$
\begin{gathered}
\left(\int_{a}^{b} \frac{\omega(r) r^{n-1}}{(1+r)^{(n-1) p / 2}} d r\right)^{1 / p}\left(\int_{a}^{b} \frac{\omega(r)^{-p^{\prime} / p} r^{n-1}}{(1+r)^{(n-1) p^{\prime} / 2}} d r\right)^{1 / p^{\prime}} \\
<K \int_{a}^{b}\left(\frac{r}{1+r}\right)^{n-1} d r
\end{gathered}
$$

whenever $0 \leqslant a<b<\infty$. Then there is a constant $C$ depending only on $n, p$ and $K$ such that

$$
\int_{\mathbf{R}^{n}}\left|\left(T_{n} f\right)(x)\right|^{p} \omega(|x|) d x<C \int_{\mathbf{R}^{n}}|f(x)|^{p} \omega(|x|) d x
$$

for every radial function $f \in L^{p}\left(\mathbf{R}^{n}, \omega(|x|)\right)$.

Taking $\omega(r)=r^{\alpha}$, it is an elementary exercise to check that $\omega(r)$ satisfies $(1.1)$ if and only if $-n+(n-1) p / 2<\alpha<-n+(n+1) p / 2$. If $f(x)$ is the characteristic function of $\left\{x \in \mathbf{R}^{n}:|x|<\varepsilon\right\}, \varepsilon>0$ small, then $\left|\left(T_{n} f\right)(x)\right|$ behaves essentially like $c_{e}|x|^{-(n+1) / 2}$ as $|x| \rightarrow \infty$ so that (1.2) applied to this $f$ shows that $\alpha<-n+$ $(n+1) p / 2$. A simple duality argument then shows that $\alpha>-n+(n-1) p / 2$ is also necessary for (1.2) to hold. Thus we have the following corollary.

COROLlaRY 1. If $\omega(r)=r^{\alpha}$ then (1.2) holds if and only if $-n+(n-1) p / 2<\alpha$ $<-n+(n+1) p / 2$.

In particular, if we set $\alpha=0$ in Corollary 1 we recover the result of Herz cited above.

It may also be noted that when $n=1$ the condition (1.1) is the $\mathrm{A}_{p}$ condition of Muckenhoupt [7] applied to $\omega(r)$ considered as an even function on $\mathbf{R}$. The $A_{p}$ condition is known to be both necessary and sufficient for (1.2) when $n=1$.

By considering separately the three fundamental cases $0=a<b<1,1=a<$ $b, 0<a<b \leqslant 2 a$, it is easy to see that if $\omega$ satisfies (1.1) for $n=1, p=2$, then $\omega$ satisfies (1.1) for all $n>1, p=2$ (with a new constant $K$ ). Thus we have Corollary 2.

COROLlaRY 2. If $p=2$ and $\omega(r)$ considered as an even function on $\mathbf{R}$ satisfies Muckenhoupt's $\mathrm{A}_{2}$ condition, then (1.2) holds.

On the other hand, if $n>1$ there are $\omega$ which satisfy (1.1) but do not satisfy the $A_{p}$ condition. Corollary 1 provides examples for this if $p \neq 2$ since $\omega(r)=r^{\alpha}$ satisfies the $A_{p}$ condition if and only if $-1<\alpha<p-1$, while the example $\omega(r)=r^{\alpha}(1+r)^{\beta-\alpha},-1<\beta<1,-n<\alpha<n$, satisfies (1.1) with $p=2$ but satisfies the $\mathrm{A}_{2}$ condition only if $-1<\beta<1,-1<\alpha<1$.

If $P_{k}(x)$ is a homogeneous harmonic polynomial in $\mathbf{R}^{n}$ of degree $k$ and $f_{0}(|x|) P_{k}(x) \in L^{2}\left(\mathbf{R}^{n}\right)$ it is well known [9, Chapter IV, §2] that

$$
\left(T_{n} f_{0} P_{k}\right)(x)=(-1)^{k}\left(R_{n+2 k} f_{o}\right)(|x|) P_{k}(x)
$$

so we have the following generalization of Theorem 1 . 
Theorem 2. Let $1<p<\infty, 1 / p+1 / p^{\prime}=1$ and let $P_{k}(x)$ be a homogeneous harmonic polynomial in $\mathbf{R}^{n}$ of degree $k$. Suppose there is a constant $K$ such that the nonnegative function $\omega(r)$ defined on $(0, \infty)$ satisfies

$$
\begin{gathered}
\left(\int_{a}^{b} \frac{\omega(r) r^{k p+n-1}}{(1+r)^{(n+2 k-1) p / 2}} d r\right)^{1 / p}\left(\int_{a}^{b} \frac{\omega(r)^{-p^{\prime} / p} r^{k p^{\prime}+n-1}}{(1+r)^{(n+2 k-1) p^{\prime} / 2}} d r\right)^{1 / p^{\prime}} \\
<K \int_{a}^{b}\left(\frac{r}{1+r}\right)^{n+2 k-1} d r
\end{gathered}
$$

for all $0<a<b<\infty$. Then there is a constant $C$ depending only on $n+2 k$, $p$ and K such that

$$
\int_{\mathbf{R}^{n}}\left|\left(T_{n} f P_{k}\right)(x)\right|^{p} \omega(|x|) d x<C \int_{\mathbf{R}^{n}}\left|f(x) P_{k}(x)\right|^{p} \omega(|x|) d x
$$

for every radial function $f$ with $f P_{k} \in L^{p}\left(\mathbf{R}^{n}, \omega(|x|)\right)$.

Theorem 2 is perhaps of particular interest when $p=2$ because of its relation to a problem of E. Stein [8, Problem 5]. If $n$ is fixed and $\omega$ satisfies the $A_{2}$ condition, then Corollary 2 shows that (1.4) holds for all $k>0$ with $C$ depending on $k$. If it could be shown that $C$ is bounded as a function of $k$, the well-known decomposition of $L^{2}\left(R^{n}\right)$ in terms of spherical harmonics [9, Chapter IV, \$2] would show that $T_{n}$ is bounded in $L^{2}\left(R^{n}, \omega(|x|)\right)$ whenever $\omega$ satisfies the $A_{2}$ condition. This would be consistent with Hirschman's result cited above, and while our methods do not show it, we conjecture that this is indeed the case.

2. Lemmas. The proof of Theorem 1 depends on several results from [1] and [6] as well as a representation of $R_{n}$ given in [5]. These are stated here for convenience of reference.

$$
\text { If } f(x)=f_{0}(|x|), x \in \mathbf{R}^{n} \text {, then }\left(T_{n} f\right)(x)=\left(R_{n} f_{0}\right)(|x|) \text { where }
$$

$$
\left(R_{n} g\right)(s)=\int_{0}^{\infty} K_{n}(s, r) g(r) d r, \quad s>0
$$

with

$$
K_{n}(s, r)=\frac{2 \pi s(r / s)^{n / 2}}{s^{2}-r^{2}}\left\{s J_{(n-2) / 2}(2 \pi r) J_{n / 2}(2 \pi s)-r J_{(n-2) / 2}(2 \pi s) J_{n / 2}(2 \pi r)\right\}
$$

and $J_{\nu}$ is the Bessel function of order $\nu$ of the first kind.

For the remainder of this section let $1<p<\infty, 1 / p+1 / p^{\prime}=1$ and let $U(r)$, $V(r)$ denote nonnegative functions defined on $(0, \infty)$.

Lemma 1 [1, Lemma 1]. Suppose there is a constant $K$ such that

$$
\left(\int_{a}^{b} V(r) d r\right)^{1 / p}\left(\int_{a}^{b} V(r)^{-p^{\prime} / p} d r\right)^{1 / p^{\prime}} \leqslant K|b-a|
$$

whenever $0<a<b \leqslant 2 a$. Then there is a constant $C$ depending only on $p$ and $K$ such that

$$
\int_{0}^{\infty} \mid \text { p.v. }\left.\int_{s / 2}^{2 s} \frac{f(r)}{s-r} d r\right|^{p} V(s) d s<C \int_{0}^{\infty}|f(r)|^{p} V(r) d r
$$


LEMMA 2 ([6], [1]). Suppose there is a constant $A$ such that

$$
\left(\int_{a}^{\infty} U(r) d r\right)^{1 / p}\left(\int_{0}^{a} V(r)^{-p^{\prime} / p} d r\right)^{1 / p^{\prime}}<A
$$

for all $a>0$. Then

$$
\int_{0}^{\infty}\left|\int_{0}^{s} f(r) d r\right|^{p} U(s) d s<p\left(p^{\prime}\right)^{p-1} A^{p} \int_{0}^{\infty}|f(r)|^{p} V(r) d r
$$

The next lemma was proved in [1, Lemma 2] for the special case $a_{0}=0$, but an examination of the proof shows that it carries over without significant change to the present situation $a_{0} \geqslant 0$.

LEMMA 3. Suppose $a_{0} \geqslant 0$ and that for some $\varepsilon>0$ there is a constant $K$ such that

$$
\left(\int_{a}^{\infty}\left(\frac{a}{r}\right)^{e} \frac{V(r)}{r^{p}} d r\right)^{1 / p}\left(\int_{0}^{a} V(r)^{-p^{\prime} / p} d r\right)^{1 / p^{\prime}} \leqslant K
$$

for all $a>a_{0}$. Then there is a constant $K_{1}$ depending only on $p, \varepsilon$ and $K$ such that

$$
\left(\int_{a}^{\infty} \frac{V(r)}{r^{p}} d r\right)^{1 / p}\left(\int_{0}^{a} V(r)^{-p^{\prime} / p} d r\right)^{1 / p^{\prime}}<K_{1}
$$

for all $a>a_{0}$.

3. Proof of Theorem 1. The proof will be accomplished by proving the three inequalities

$$
\int_{0}^{\infty}\left|\left(R_{n, i} f\right)(s)\right|^{p} \omega(s) s^{n-1} d s \leqslant C \int_{0}^{\infty}|f(r)|^{p} \omega(r) r^{n-1} d r, \quad i=1,2,3,
$$

where

$$
\begin{gathered}
\left(R_{n, i} f\right)(s)=\int_{0}^{\infty} K_{n, i}(s, r) f(r) d r \\
K_{n, 1}(s, r)= \begin{cases}\left|K_{n}(s, r)\right| & \text { if } r<s / \sqrt{2} \\
0 & \text { otherwise; }\end{cases} \\
K_{n, 3}(s, r)= \begin{cases}\left|K_{n}(s, r)\right| & \text { if } r>\sqrt{2} s, \\
0 & \text { otherwise; }\end{cases} \\
K_{n, 2}(s, r)= \begin{cases}K_{n}(s, r) & \text { if } s / \sqrt{2}<r<\sqrt{2} s, \\
0 & \text { otherwise; }\end{cases}
\end{gathered}
$$

and $K_{n}(s, r)$ is as given by (2.1).

Consider $R_{n, 2}$ first. The change of variable $r^{2}=t$ shows that $\left(R_{n, 2} f\right)(s)$ is the difference of

$$
\left(R_{n, 2,1} f\right)(s)=\frac{\pi J_{n / 2}(2 \pi s)}{s^{(n-4) / 2}} \int_{s^{2} / 2}^{2 s^{2}} \frac{J_{(n-2) / 2}(2 \pi \sqrt{t}) t^{(n-2) / 4}}{s^{2}-t} f(\sqrt{t}) d t
$$

and

$$
\left(R_{n, 2,2} f\right)(s)=\frac{\pi J_{(n-2) / 2}(2 \pi s)}{s^{(n-2) / 2}} \int_{s^{2} / 2}^{2 s^{2}} \frac{J_{n / 2}(2 \pi \sqrt{t}) t^{n / 4}}{s^{2}-t} f(\sqrt{t}) d t
$$


Now, from (1.1) it follows that

$$
\left(\int_{a}^{b} \omega(r) d r\right)^{1 / p}\left(\int_{a}^{b} \omega(r)^{-p^{\prime} / p} d r\right)^{1 / p^{\prime}}<2^{n-1} K|b-a|
$$

whenever $0<a<b<\sqrt{2} a$, and hence the change of variable $r^{2}=t$ shows

$$
\left(\int_{a^{2}}^{b^{2}} \omega(\sqrt{t}) \frac{d t}{\sqrt{t}}\right)^{1 / p}\left(\int_{a^{2}}^{b^{2}} \omega(\sqrt{t})^{-p^{\prime} / p} \frac{d t}{\sqrt{t}}\right)^{1 / p^{\prime}}<2^{n} K|b-a|
$$

whenever $b^{2} \leqslant 2 a^{2}$. It is now easy to see that there is a constant $C$ depending on $n$ and $p$ such that

$$
\begin{gathered}
\left(\int_{a^{2}}^{b^{2}} \frac{t^{p+n / 2-1}}{(1+t)^{(n+1) p / 4}} \omega(\sqrt{t}) d t\right)^{1 / p}\left[\int_{a^{2}}^{b^{2}}\left(\frac{t^{p+n / 2-1}}{(1+t)^{(n+1) p / 4}} \omega(\sqrt{t})\right)^{-p^{\prime} / p} d t\right]^{1 / p^{\prime}} \\
<C K b|b-a|<C K\left|b^{2}-a^{2}\right|
\end{gathered}
$$

whenever $b^{2} \leqslant 2 a^{2}$. Thus $V(r)=r^{p+n / 2-1}(1+r)^{-(n+1) p / 4} \omega(\sqrt{r})$ satisfies (2.2). The variable change $s^{\prime}=s^{2}$ and the estimate (see [10, pp. 49, 199])

$$
\left|J_{\nu}(t)\right|<c_{\nu} \frac{t^{\nu}}{(1+t)^{\nu+1 / 2}}
$$

followed by an application of Lemma 1 and a further appeal to (3.2) then shows that

$$
\begin{aligned}
\int_{0}^{\infty}\left|\left(R_{n, 2,1} f\right)(s)\right|^{p} \omega(s) s^{n-1} d s \\
\quad=\int_{0}^{\infty}\left|\frac{\pi J_{n / 2}(2 \pi \sqrt{s})}{s^{(n-4) / 4}} \int_{s / 2}^{2 s} \frac{J_{(n-2) / 2}(2 \pi \sqrt{t}) t^{(n-2) / 4}}{s-t} f(\sqrt{t}) d t\right|^{p} \frac{\omega(\sqrt{s})}{2} s^{n / 2-1} d s \\
\quad<C \int_{0}^{\infty}\left|\int_{s / 2}^{2 s} \frac{J_{(n-2) / 2}(2 \pi \sqrt{t}) t^{(n-2) / 4}}{s-t} f(\sqrt{t}) d t\right|^{p} V(s) d s \\
\quad<C \int_{0}^{\infty}\left|J_{(n-2) / 2}(2 \pi \sqrt{t}) t^{(n-2) / 4} f(\sqrt{t})\right|^{p} V(t) d t \\
\quad<C \int_{0}^{\infty}|f(r)|^{p} r^{n-1} \omega(r) d r
\end{aligned}
$$

for suitable constant $C$ depending on $n, p, K$, not necessarily the same at each occurrence. A similar derivation applies to $R_{n, 2,2}$. This proves (3.1) for $i=2$.

Now consider $R_{n, 1}$. Because of (3.2) we have

$$
\begin{aligned}
K_{n, 1}(s, r) & <C \frac{r^{n / 2}}{s^{(n-2) / 2}\left|s^{2}-r^{2}\right|}\left\{\frac{r^{n / 2-1} s^{n / 2+1}}{(1+r)^{(n-1) / 2}(1+s)^{(n+1) / 2}}\right. \\
& \left.<C \frac{r^{n / 2+1} s^{n / 2-1}}{(1+r)^{(n+1) / 2}(1+s)^{(n-1) / 2}}\right\} \\
& +\frac{r^{n-1}}{(1+r)^{(n-1) / 2}(1+s)^{(n+1) / 2}}
\end{aligned}
$$


for suitable constants $C$ depending only on $n$, where to obtain the last inequality we used $r<s / \sqrt{2}$ whenever $K_{n, 1}(s, r) \neq 0$. Thus,

$$
\left|\left(R_{n, 1} f\right)(s)\right| \leqslant \frac{C}{(1+s)^{(n+1) / 2}} \int_{0}^{s} \frac{r^{n-1}}{(1+r)^{(n-1) / 2}}|f(r)| d r
$$

and hence (3.1) for $i=1$ will follow from Lemma 2 if we show that (1.1) implies

$$
\left(\int_{a}^{\infty} \frac{\omega(s) s^{n-1}}{(1+s)^{(n+1) p / 2}} d s\right)\left(\int_{0}^{a} \frac{\omega(s)^{-p^{\prime} / p} s^{n-1}}{(1+s)^{(n-1) p^{\prime} / 2}} d s\right)^{p-1}<C
$$

for all $a>0$ with $C$ depending only on $p, n$ and $K$. To show this, note that (1.1) leads to

$$
\left(\int_{a}^{t} \frac{\omega(r) r^{n-1}}{(1+r)^{(n-1) p / 2}} d r\right)\left(\int_{0}^{a} \frac{\omega(r)^{-p^{\prime} / p} r^{n-1}}{(1+r)^{(n-1) p^{\prime} / 2}} d r\right)^{p-1}<K^{p}\left(\int_{0}^{t}\left(\frac{r}{1+r}\right)^{n-1} d r\right)^{p}
$$

whenever $0<a<t$. Multiplying this inequality by $(1+t)^{-p-2}$, integrating the result over $t>a$, then applying Fubini's theorem on the left side shows that

$$
\begin{aligned}
& \left(\int_{a}^{\infty}\left(\frac{1+a}{1+r}\right) \frac{\omega(r) r^{n-1}}{(1+r)^{(n+1) p / 2}} d r\right)\left(\int_{0}^{a} \frac{\omega(r)^{-p^{\prime} / p} r^{n-1}}{(1+r)^{(n-1) p^{\prime} / 2}} d r\right)^{p-1} \\
& \quad<(p+1) K^{p}(1+a) \int_{a}^{\infty} \frac{1}{(1+t)^{2}}\left(\frac{1}{1+t} \int_{0}^{t}\left(\frac{r}{1+r}\right)^{n-1} d r\right)^{p} d t \\
& \quad<(p+1) K^{p}(1+a) \int_{a}^{\infty} \frac{d t}{(1+t)^{2}}=(p+1) K^{p}
\end{aligned}
$$

Except for the factor $(1+a) /(1+r)$ in the left integral this is (3.3). To see that this factor may be deleted we consider separately the cases $a<1$ and $a>1$. If $a>a_{0}=1,(3.4)$ is clearly equivalent to

$$
\left(\int_{a}^{\infty}\left(\frac{a}{r}\right) \frac{\omega(r)}{r^{p}} \frac{(1+r)^{(n-1) p / 2}}{r^{(n-1)(p-1)}} d r\right)^{1 / p}\left(\int_{0}^{a} \frac{\omega(r)^{-p^{\prime} / p} r^{n-1}}{(1+r)^{(n-1) p^{\prime} / 2}} d r\right)^{1 / p^{\prime}}<C K
$$

for a suitable constant $C$ depending on $p$ and $n$, and then Lemma 3 applied with $\varepsilon=1, V(r)=\omega(r)(1+r)^{(n-1) p / 2} r^{-(n-1)(p-1)}$ shows that the factor $a / r$ may be deleted provided $C K$ is replaced by a new constant $K_{1}$ depending on $K, n$, and $p$. The result is then equivalent to (3.3). Now if $a<a_{0}=1$ the left side of (3.3) is bounded by the sum of

$$
\left(\int_{a}^{1} \frac{\omega(r) r^{n-1}}{(1+r)^{(n+1) p / 2}} d r\right)\left(\int_{0}^{1} \frac{\omega(r)^{-p^{\prime} / p} r^{n-1}}{(1+r)^{(n-1) p^{\prime} / 2}} d r\right)^{p-1}
$$

and

$$
\left(\int_{1}^{\infty} \frac{\omega(r) r^{n-1}}{(1+r)^{(n+1) p / 2}} d r\right)\left(\int_{0}^{1} \frac{\omega(r)^{-p^{\prime} / p} r^{n-1}}{(1+r)^{(n-1) p^{\prime} / 2}} d r\right)^{p-1}
$$


That the first of these has the required bound follows directly from (1.1) while the boundedness of the second is merely the case $a=1$ of (3.3) which we have already proved. This completes the proof of (3.3) and hence also of (3.1) for $i=1$.

Finally, consider $R_{n, 3}$. The desired inequality could be proved in a manner similar to that used to deal with $R_{n, 1}$ but a proof by duality is more convenient. Note that if $\omega(r)$ satisfies $(1.1)$ for some $p$, then $\omega(r)^{-p^{\prime} / p}$ satisfies $(1.1)$ for $p^{\prime}$. Since we have proved (3.1) for $i=1$ it then follows that

$$
\int_{0}^{\infty}\left|\left(R_{n, 1} f\right)(s)\right|^{p^{\prime}} \omega(s)^{-p^{\prime} / p_{s} n-1} d s<C \int_{0}^{\infty}|f(r)|^{p^{\prime}} \omega(r)^{-p^{\prime} / p} r^{n-1} d r
$$

whenever (1.1) holds. Let $g(s) \geqslant 0$. Fubini's Theorem shows that

$$
\begin{aligned}
\int_{0}^{\infty}\left|\left(R_{n, 3} f\right)(s)\right| g(s) \omega(s) s^{n-1} d s & \leqslant \int_{0} g(s) \omega(s) s^{n-1} d s \int_{\sqrt{2}}^{\infty}\left|K_{n}(s, r)\right||f(r)| d r \\
& =\int_{0}^{\infty}|f(r)|\left(R_{n, 1} g \omega\right)(r) r^{n-1} d r .
\end{aligned}
$$

Applying Hölder's inequality we see that this is bounded by

$$
\left(\int_{0}^{\infty}|f(r)|^{p} \omega(r) r^{n-1} d r\right)^{1 / p}\left(\int_{0}^{\infty}\left|\left(R_{n, 1} g \omega\right)(r)\right|^{p^{\prime}} \omega(r)^{-p^{\prime} / p_{r} n-1} d r\right)^{1 / p^{\prime}}
$$

and then (3.5) shows that this in turn is bounded by

$$
\left(\int_{0}^{\infty}|f(r)|^{p} \omega(r) r^{n-1} d r\right)^{1 / p}\left(C \int_{0}^{\infty}|g(s)|^{p^{\prime}} \omega(s) s^{n-1} d s\right)^{1 / p^{\prime}} .
$$

The converse of Hölder's inequality now yields (3.1) for $i=3$. This completes the proof of Theorem 1.

\section{REFERENCES}

1. K. F. Andersen and B. Muckenhoupt, Weighted weak type Hardy inequalities with applications to Hilbert transforms and maximal functions, Studia Math. (to appear).

2. C. Fefferman, The multiplier problem for the ball, Ann. of Math. 94 (1971), 330-336.

3. C. Herz, On the mean inversion of Fourier and Hankel transforms, Proc. Nat. Acad. Sci. U.S.A. 40 (1954), 996-999.

4. I. I. Hirschman, Multiplier transformations. II, Duke Math. J. 28 (1961), 45-56.

5. C. E. Kenig and P. Tomas, The weak behaviour of spherical means, Proc. Amer. Math. Soc. 78 (1980), 48-50.

6. B. Muckenhoupt, Hardy's inequalities with weights, Studia Math. 44 (1972), 31-38.

7. Weighted norm inequalities for the Hardy maximal function, Trans. Amer. Math. Soc. 165 (1972), 207-226.

8. E. M. Stein, Some problems in harmonic analysis, Proc. Sympos. Pure Math., vol. 35, part I, Amer. Math. Soc., Providence, R.I., 1979.

9. E. Stein and G. Weiss, Introduction to Fourier analysis on euclidean spaces, Princeton Univ. Press, Princeton, N.J., 1971.

10. G. N. Watson, Theory of Bessel functions, Cambridge Univ. Press, London, 1966.

Department of Mathematics, University of Alberta, Edmonton, Alberta, Canada t6G 2 G1 